\title{
HDAC9 complex inhibition improves smooth muscle-dependent stenotic vascular disease
}

\author{
Christian L. Lino Cardenas, ${ }^{1}$ Chase W. Kessinger, ${ }^{1}$ Elizabeth Chou, ${ }^{2}$ Brian Ghoshhajra, ${ }^{3}$ \\ Ashish S. Yeri, ${ }^{1}$ Saumya Das, ${ }^{1}$ Neal L. Weintraub, ${ }^{4}$ Rajeev Malhotra, ${ }^{1}$ Farouc A. Jaffer, ${ }^{1}$ \\ and Mark E. Lindsay ${ }^{1,2,5,6}$ \\ ${ }^{1}$ Cardiovascular Research Center, Cardiology Division, Department of Medicine, ${ }^{2}$ Division of Vascular and Endovascular \\ Surgery, Department of Surgery, and ${ }^{3}$ Department of Radiology, Massachusetts General Hospital, Harvard Medical School, \\ Boston, Massachusetts, USA. ${ }^{4}$ Medical College of Georgia, Augusta, Georgia, USA. ${ }^{5}$ Cardiovascular Genetics Program, \\ Cardiology Division, Department of Medicine, and ${ }^{6}$ Pediatric Cardiology, Department of Pediatrics, Massachusetts Ceneral \\ Hospital, Harvard Medical School, Boston, Massachusetts, USA.
}

Patients with heterozygous missense mutations in the ACTA2 or MYH11 gene are known to exhibit thoracic aortic aneurysm and a risk of early-onset aortic dissection. However, less common phenotypes involving arterial obstruction are also observed, including coronary and cerebrovascular stenotic disease. Herein we implicate the HDAC9 complex in transcriptional silencing of contractile protein-associated genes, known to undergo downregulation in stenotic lesions. Furthermore, neointimal formation was inhibited in HDAC9- or MALAT1-deficient mice with preservation of contractile protein expression. Pharmacologic targeting of the HDAC9 complex through either MALAT1 antisense oligonucleotides or inhibition of the methyltransferase EZH2 (catalytic mediator recruited by the HDAC9 complex) reduced neointimal formation. In conclusion, we report the implication of the HDAC9 complex in stenotic disease and demonstrate that pharmacologic therapy targeting epigenetic complexes can ameliorate arterial obstruction in an experimental system.

Conflict of interest: CLLC, RM, and MEL will be filling a provisional patent on the use of inhibitors of the HDAC9 complex for the treatment of stenotic vascular disease.

License: Copyright 2019, American Society for Clinical Investigation.

Submitted: September 5, 2018 Accepted: December 13, 2018 Published: January 24, 2019

\section{Reference information:} JCI Insight. 2019;4(2):e124706 https://doi.org/10.1172/jci. insight.124706.

\section{Introduction}

In diverse forms of vascular disease, smooth muscle cells (SMCs) are known to modulate cellular phenotype typified by downregulation of SMC-restricted contractile-protein genes, and upregulate groups of genes involved in secretion of extracellular matrix, proliferation, and migration. This cellular behavior has been noted in important human diseases such as atherosclerosis, pulmonary hypertension, aortic and peripheral aneurysms, and restenosis after percutaneous arterial intervention $(1,2)$. Interestingly, the phenotype of thoracic aortic aneurysm (TAA) has been associated with human mutations in genes encoding these same SMC-restricted contractile proteins. For instance, mutations in ACTA2, the gene encoding $\alpha$-smooth muscle actin ( $\alpha$-SMA), are a major cause of nonsyndromic TAA (3). Similarly, mutations in the gene MYH11, encoding smooth muscle myosin heavy chain (smMHC), the binding partner of this actin isoform, causes a combined presentation of TAA and patent ductus arteriosis (4). On the basis of these observations and others, disruption of SMC contraction has been hypothesized as an underlying mechanism of TAA pathogenesis (5). However, in addition to extensive implication of the smooth muscle contractile dysfunction in aneurysmal disease, SMCs have also been extensively implicated in stenotic/ischemic vascular disease. In fact, individuals with ACTA2 mutations demonstrate pathologies such as coronary, pulmonary, or intracranial arterial stenosis $(6,7)$. MYH11 mutations have similarly been shown to cause a moyamoya-like vascular pathology, implicating this gene family in stenotic as well as aneurysmal disorders in humans (8). At the center of SMC phenotype modulation observed in vascular disease is a complex network of transcriptional pathways that coordinate repression of contractile elements such as $\alpha$-SMA and smMHC and upregulation of synthetic and proliferative function $(2,9)$.

Recently, we described one such pathway active in TAA pathogenesis consisting of the histone deacetylase, HDAC9, the chromatin remodeling protein Brahma-related gene 1 (BRG1), and the long noncoding RNA (lncRNA), MALAT1 (10). This HDAC9 chromatin-modifying complex is recruited to the promoters 
of vascular SMC-specific (VSMC-specific) genes in the presence of gene products modified with TAA-associated mutations. Among other functions, the HDAC9 complex recruits Polycomb repressive complex 2 (PRC2) to catalyze the trimethylation of histone 3 on lysine 27 (H3K27) through EZH2, its enzymatic subunit. Inhibition of the HDAC9 complex slowed aneurysm progression in conjunction with decreased H3K27 trimethylation and the restoration of contractile protein expression $(10,11)$. Genetic evidence in humans implicates the HDAC9 locus in the pathogenesis of not only aneurysms, but also ischemic arterial disease such as coronary artery disease and large vessel ischemic stroke $(12,13)$. We therefore hypothesized that the HDAC9 complex may also be involved in transcriptional control of SMCs in forms of vascular disease also involving arterial stenosis.

In this study, we investigated the role of the HDAC9 complex in modulating stenotic vascular disease using the murine carotid artery ligation model. We find evidence of HDAC9 activation in medial and neointimal SMCs after ligation and binding of the HDAC9-containing complex to SMC contractile-protein promoters correlated with transcriptional downregulation of contractile elements. Nonbiased chromatin immunoprecipitation sequencing assays demonstrate multiple targets of the HDAC 9 complex including cGMP, angiotensin, adrenergic, and oxytocin signaling pathways. Utilizing these data, we investigated inhibition of the HDAC9 pathway as a therapeutic modality. In experimental animals, inhibition of MALAT1 or HDAC9 expression arrested neointimal hyperplasia and partially normalized the transcriptional signature of the artery. Pharmacologic targeting of the HDAC9 complex with GapmeR antisense oligonucleotides against MALAT1 or small-molecule inhibitor GSK343 was effective at controlling arterial stenosis. These data illuminate a critical role in HDAC9-mediated transcriptional silencing in the process of stenotic arterial disease and validate this pathway as a target for stenotic arterial disease.

\section{Results}

Identification of HDAC9 targets in cells exposed to the R179H mutant allele of ACTA2. We previously identified an epigenetic remodeling complex activated in VSMCs (HDAC9-BRG1-MALAT1) under the influence of genetic mutations that cause aortic aneurysm (10). However, some forms of genetically triggered aortic disease (GTAD) such as those caused by mutations in the ACTA2 or MYH11 gene also cause stenotic disease $(6,8)$. In particular, recurrent missense mutations in the codon for arginine 179 causes a severe form of smooth muscle dystrophy with both vascular aneurysms and stenoses (Figure 1A) (7). To gain insight into genetic targets that may explain this phenotypic divergence, we performed whole-genome chromatin immunoprecipitation of HDAC9, BRG1, and the histone mark histone 3 trimethylated lysine 27 (H3K27me3) followed by next-generation sequencing analysis (ChIP-seq) within VSMCs transduced with an ACTA2 ${ }^{\mathrm{R} 179 \mathrm{H}}$ allele. Analysis of genes enriched in cells expressing ACTA2 ${ }^{\mathrm{R} 179 \mathrm{H}}$ were sought by subtracting genes identified in baseline conditions. The results demonstrate 1,141 genes identified by each of these 3 separate interactions (Figure 1, B and C, and Supplemental Table 1; supplemental material available online with this article; https://doi.org/10.1172/jci.insight.124706DS1). In silico analysis of the core genes implicates several pathways involved in the modulation of VSMC force generation including cGMPPKG1, renin, and adrenergic signaling in addition to genes comprising the VSMC contraction apparatus (Figure 1C and Supplemental Table 2). Interestingly, the ACTA2 locus itself demonstrated substantial enrichment of H3K27me3 as well as association with HDAC9 and BRG1 (Figure 1B). This regulation of the ACTA2 locus appears to be specific, as other alleles known to induce aneurysmal but not stenotic disease, such as TGFBR2 ${ }^{\mathrm{G} 357 \mathrm{~W}}$, fail to suppress the ACTA2 locus (Supplemental Figure 1). Applying multiple comparisons correction to our ChIP-seq data reveals $A C T A 2$ as the only gene locus at genome-wide significance (Supplemental Figure 1). To determine if this silencing was mediated by the HDAC9 complex we assayed for H3K27me3 modifications by targeted ChIP-qPCR at the ACTA2 promoter and found them to be MALAT1 dependent (Figure 1D).

The HDAC9 complex in murine experimental neointimal hyperplasia and arterial stenosis. To gain insight into the role of epigenetic silencing of gene products (such as $\alpha$-SMA) in vascular stenosis we performed murine carotid arterial ligation. Unilateral ligation of the carotid artery in mice induces significant transcriptional changes in the arterial media and stimulates the formation of an extensive neointima (Figure 2A). Grossly, reactionary neointimal changes, including secretion of matrix-degrading enzymes such as MMPs, SMC proliferation and phenotypic change, and inflammation are seen. Contractile proteins such as $\alpha$-SMA and $\operatorname{Sm} 22 \alpha$ are strongly downregulated within the ligated artery, while matrix-degrading 
A

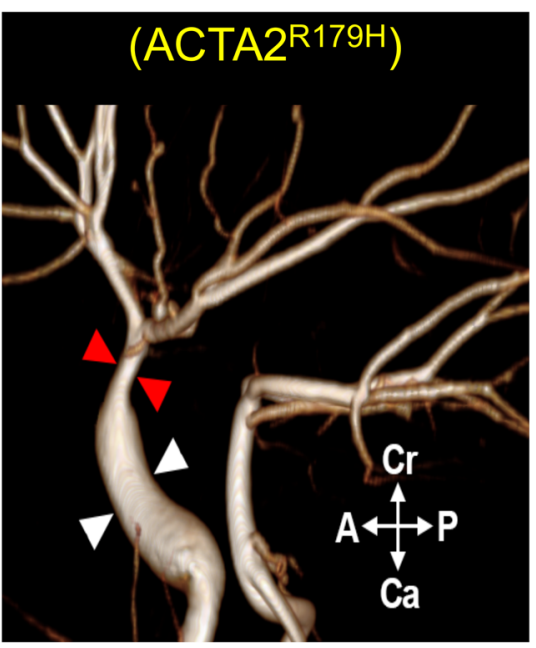

B

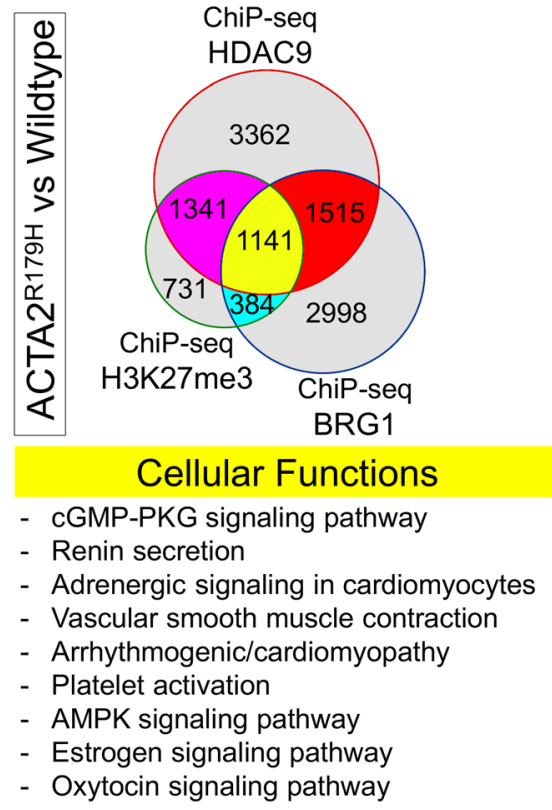

C
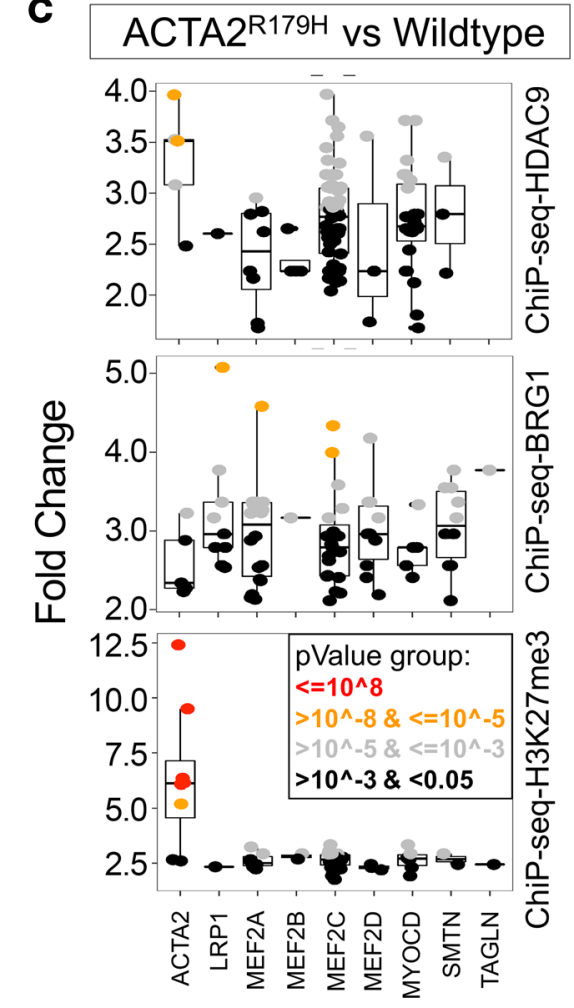

D

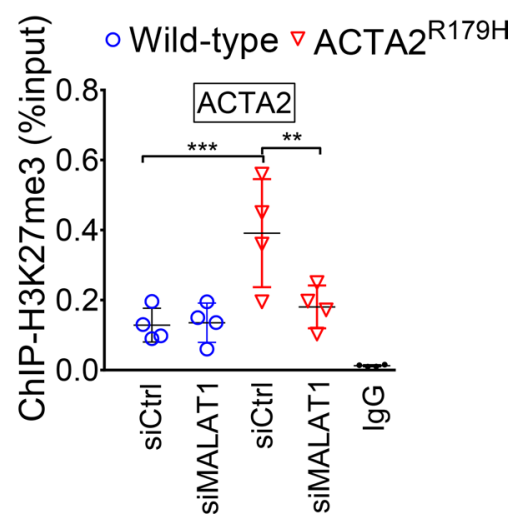

Figure 1. ACTA2 ${ }^{\mathrm{R} 179 \mathrm{H}}$ mutation induces HDAC9-BRG1-MALAT1 complex interaction with genetic loci associated with VSMC contractility. (A) Lateral view of left-sided intracranial arterial system in patient with $A C T A 2^{\mathrm{R} 179 \mathrm{H}}$ mutation. Cavernous internal carotid dilatation (white arrows) with abrupt tapering and obstructive lesion at terminus (red arrows). (B) Venn diagram describing ChIP-seq experiment in human VSMCs under conditions of ACTA2 ${ }^{\mathrm{R} 179 \mathrm{H}}$ expression shows overlapping enrichment of HDAC9, BRC1, and H3K27me3 modification at 1,141 gene loci versus wild-type cells. Pathway analysis demonstrates multiple cellular functions involved in contraction. (C) Box plots show fold-change enrichment of HDAC9, BRG1, and H3K27me3 at locus of SMC contractile elements in ACTA2 ${ }^{\mathrm{R} 17 \mathrm{H}}$ mutant versus wild-type cells. Color-coded nominal $P$ values of individual genomic sites within loci are demonstrated. $P$-value annotation of the peaks from MACS2 was performed using the ChipSeeker package in R. (D) Inhibition of MALAT1 in ACTA2 ${ }^{\mathrm{R} 79 \mathrm{H}}$ mutant cells decreased levels of the repression mark $\mathrm{H} 3 \mathrm{~K} 27 \mathrm{me} 3$ at the ACTA2 promoter. Statistical significance was determined by 1 -way ANOVA with Tukey's post hoc test (versus wild-type cells). ${ }^{* *} P<0.001,{ }^{* * *} P<0.0001$.

enzymes such as MMP9 showed increased expression (Figure 2B). Previously, we documented targeting of the HDAC9 complex to the promoters of contractile proteins in cellular models of aortic aneurysm. We therefore investigated whether this complex may mediate the observed silencing during neointimal formation within the carotid ligation model. First we investigated the expression level of the components of the complex, HDAC9, BRG1, and MALAT1, in ligated arteries. Both HDAC9 and MALAT1 mRNAs were significantly upregulated (Figure $2 \mathrm{C}$ ) and the components of the complex colocalized in ligated arteries within intimal nuclei (Figure 2, D and $\mathrm{E}$ ). 
Inhibition of the HDAC9 complex improves neointimal hyperplasia. The ability of MALAT1 inhibition to prevent accumulation of $\mathrm{H} 3 \mathrm{~K} 27 \mathrm{me} 3$ chromatin marks at the ACTA2 promoter (Figure 1D) suggests that downregulation may help facilitate phenotype change necessary for neointimal hyperplasia formation. To determine if inhibition of the HDAC9 complex inhibits neointimal formation we ligated the carotid arteries of mice deficient in MALAT1 or HDAC9. To test this hypothesis, carotid artery ligation was performed on MALAT1-deficient (Malat1 ${ }^{-/-}$) (14) and VSMC-targeted HDAC9-deficient animals (Hdac $9^{\text {t/flt }}$ :Tagln-cre) (10). Supporting an important role in the formation of neointimal proliferation, Hdac9-and Malat-deficient mice showed significantly reduced neointimal hyperplasia and stenosis 21 days after ligation (Figure 3, A and B). Additionally, immunostaining of contractile elements of VSMCs typically downregulated during neointimal formation (SM22, $\alpha$-SMA, and calponin proteins) remained preserved in ligated carotid arteries

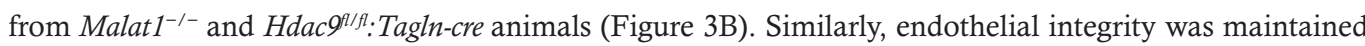
in Malat1 $^{-/-}$and Hdac $9^{\text {I/fl}}$ :Tagln-cre as assessed by CD31 staining in ligated arteries (Figure 3C). To determine the extent of transcriptional dysregulation in the carotid arteries of targeted mice, a panel of contractile and synthetic mRNAs was assayed. Among contractile gene products, genetic inhibition of the HDAC 9 complex had the most dramatic effect on the ACTA2 gene (Figure 3D). Consistent with both improvement in the stenotic phenotype and restoration of ACTA2 expression, matrix metalloproteinase 9 (MMP9) protein expression is suppressed in Malat $^{-/-}$and Hdac $9^{\text {flflf }}$ :Tagln-cre mice (Figure 3, C and D).

We next examined proliferative and remodeling pathways known to be activated during neointimal formation $(15,16)$. We therefore assayed activation of the platelet-derived growth factor receptor (PDGFR) and focal adhesion kinase (FAK) pathways by phospho-specific antibody staining. Consistent with the observed palliation of neointimal development, dramatic reduction of phosphorylation of PDGFR and

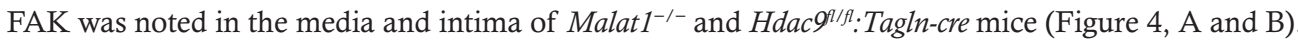

Pharmacologic inhibition of the HDAC9 complex improves stenotic pathology. The improvement of stenotic vascular phenotype in Malat ${ }^{-/-}$and Hdac $9^{\text {I/fl}}$ :Tagln-cre mice suggested that pharmacologic targeting of the complex could improve vascular performance. In previous work, we demonstrated that the HDAC9 complex recruits $\mathrm{PRC} 2$, allowing silencing modifications of histone $\mathrm{H} 3$ at contractile-protein promoters. Inhibition of the catalytic subunit of PRC2, an enzyme named EZH2, inhibited aortic aneurysm growth in mice (11). Therefore, we explored the use of 2 separate treatments to inhibit transcriptional events downstream of the HDAC9 complex. In the first, we used a GapmeR targeting the lncRNA MALAT1 to dissociate the complex that recruits PRC2 to chromatin. GapmeRs are small antisense DNAs with modified end nucleotides that recruit RNAse $\mathrm{H}$ for target transcript degradation. Using this peripherally injected GapmeR, we first assayed repression of Malat1 in arterial tissue (Figure 5A). In the second step, we used a pharmacologic inhibitor of the EZH2 enzyme, GSK343, to prevent PRC2-mediated transcriptional repression.

Treatment of animals with either GapmeR-Malat1 or GSK343 initiated before and continued during carotid artery ligation dramatically improved the extent of the stenotic response (Figure 5, B and C). Consistent with an improved gross phenotype, both GapmeR-Malat1 and GSK343 treatment improved the expression of contractile proteins such as $\alpha$-SMA and SM22 $\alpha$, typically downregulated in neointimal hyperplasia (Figure 5B). Supporting the concept of EZH2 being a primary therapeutic target in the arterial response, formation of a neointima was also inhibited in mice with SMC-specific inactivation of the EZH2

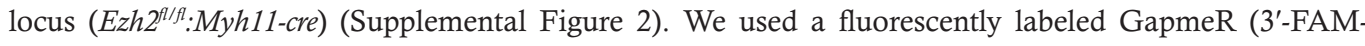
GapmeR-Malat1) to assay delivery to the vasculature. 3'-FAM-positive carotid VSMCs as well as VSMCs from GSK343-treated animals demonstrated robust expression of $\alpha$-SMA, in contrast to carotid VSMCs in wild-type animals (Figure 5D). Likewise, GapmeR-Malat1 and GSK343 treatment inhibited the activity of MMPs (Figure 5E) known to be involved in the matrix remodeling of neointimal formation.

Similar to observations in Malat1 ${ }^{-1-}$ and Hdac $9^{\text {flff }}$ :Tagln-cre mice, mice treated with GapmeR-Malat1 or GSK343 showed dramatic reduction of phosphorylation of PDGFR, and FAK was in both the media and intima of carotid arteries (Figure 6, A and B). Importantly, carotid arteries from mice treated with GapmeR-Malat1 or GSK343 also showed decreased accumulation of Hdac9 and Mmp9 in the arterial media and intima (Figure 6C).

\section{Discussion}

Stenotic as well as aneurysmal lesions have been noted in individuals with mutations in members of the smooth muscle contractile apparatus. In this study, we used ChIP to identify targets of the HDAC9 complex in cells expressing an $\mathrm{ACTA} 2^{\mathrm{R} 179 \mathrm{H}}$ allele. Interestingly, multiple loci were identified interacting with 

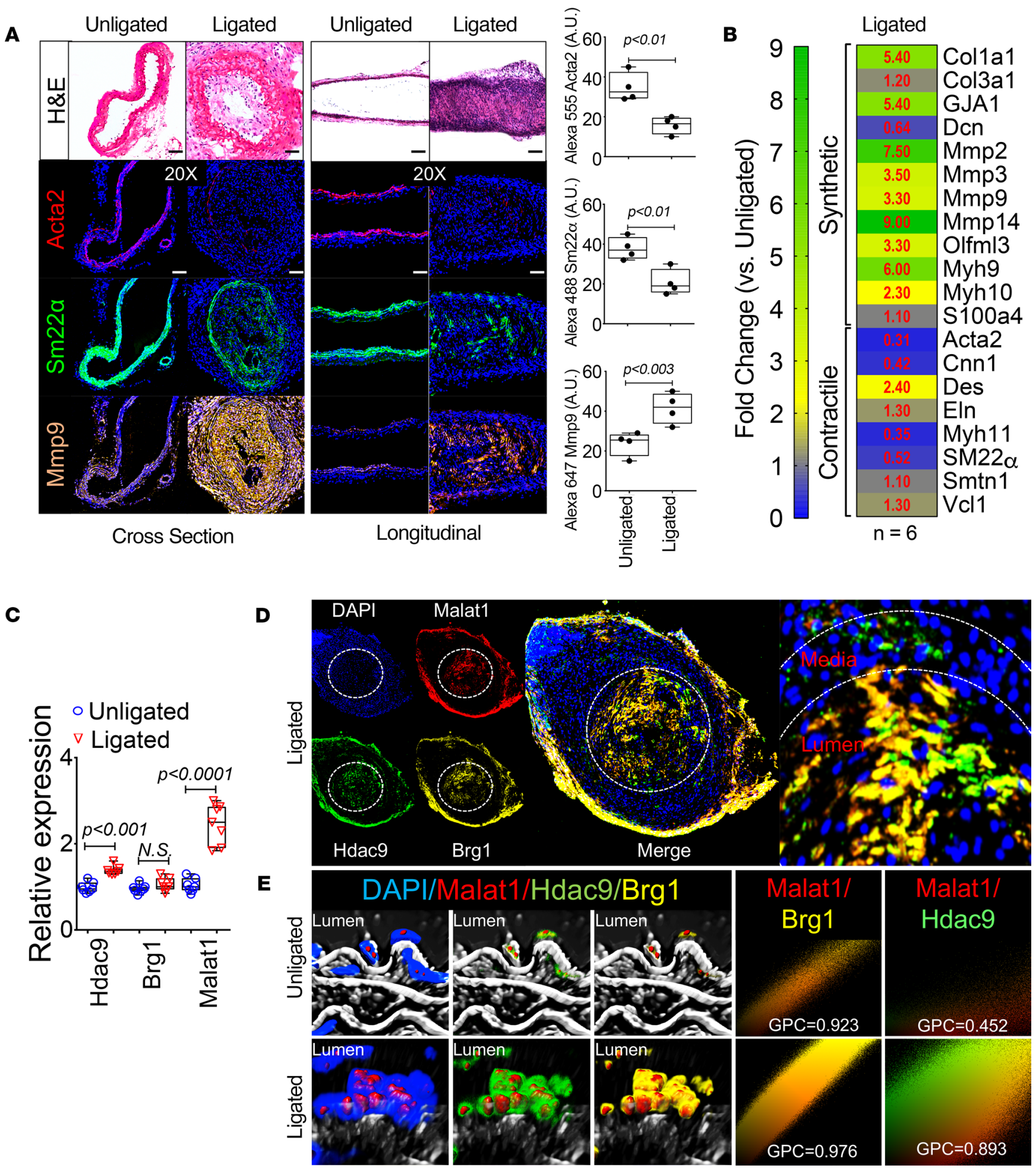

Figure 2. Experimental model of arterial stenosis induces Hdac9-Brg1-Malat1 complex formation. (A) Histological and immunofluorescence analysis of ligated and unligated carotids demonstrates neointimal formation and lumen obstruction ( $n=2$ male and 2 female). Ligated carotids show significant reduction in Acta2 ( $\alpha$-Sma, red), Sm22 $\alpha$ (green), and increased expression of Mmp9 (orange). Significance was calculated using an unpaired $t$ test (2-tailed vs. unligated carotid). The red asterisk indicates the most downregulated gene in ligated carotid samples when compared with the contralateral unligated carotid. (B) Heatmap of contractile and synthetic SMC markers from ligated carotids ( $n=3$ male and 3 female) versus unligated carotids ( $n=3$ male and 3 female) of wild-type mice. (C) RT-qPCR analysis demonstrates upregulation of Hdac9 and Malat1 mRNAs in ligated carotids of wild-type mice. Significance was calculated using an unpaired $t$ test (2-tailed, vs. unligated carotid). (D) Immunofluorescence (Hdac9 and Brg1) and in situ hybridization (Malat1) staining of ligated carotid localizes robust expression to the newly formed neointima. (E) 3D sequential immunofluorescence and in situ hybridization microscopy of ligated carotids show colocalization of Hdac9 (green), Brg1 (yellow), and Malat1 (red) in neointimal cells. Global Pearson's colocalization (CPC) of spatial overlap between Malat1-Hdac9 and Malat1-Brg1 is shown. Scale bars: $50 \mu \mathrm{m}$. 
A
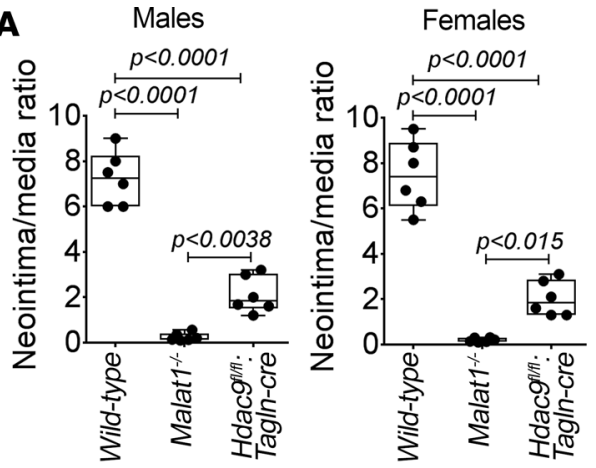

C

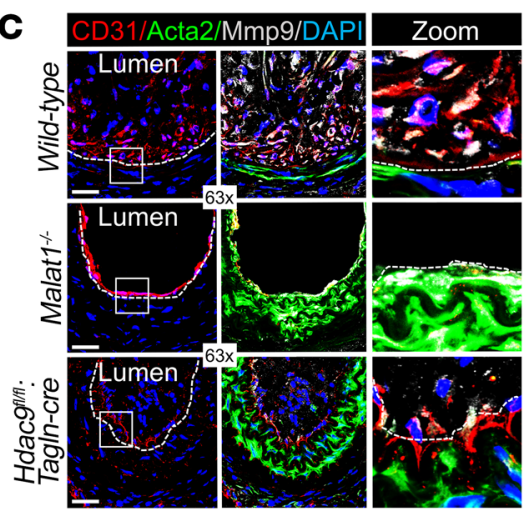

B
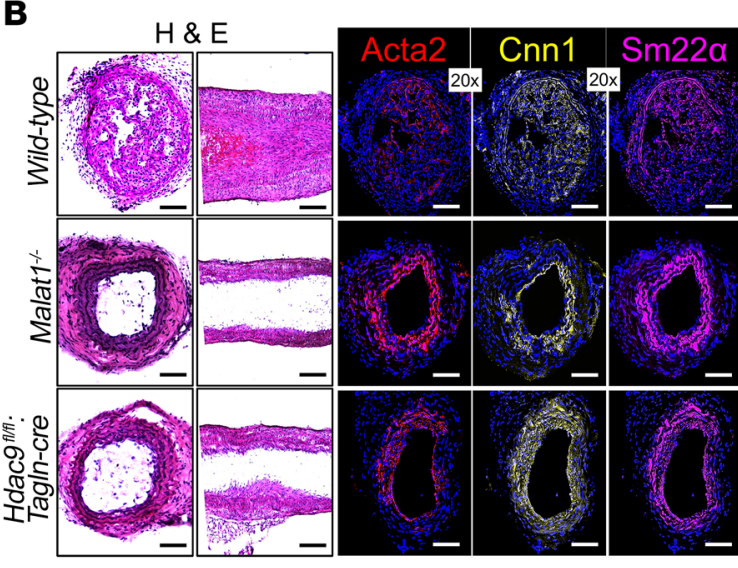

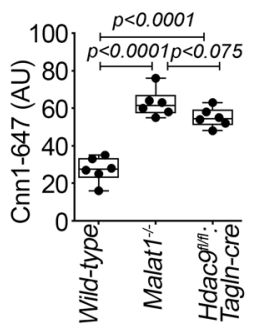

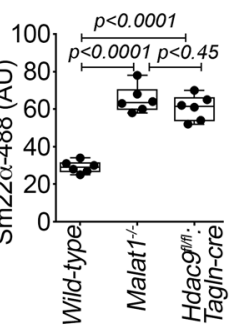
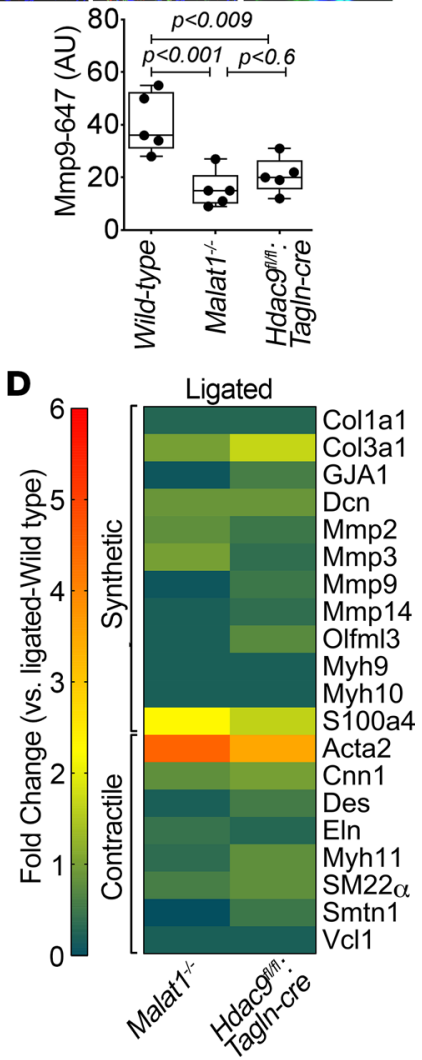

Figure 3. Genetic disruption of Hdac9 or Malat1 inhibits neointimal formation. (A) Histological quantification of neointimal hyperplasia in ligated carotid from wild-type ( $n=6$ male and 6 female), Malat1 ${ }^{-1-}(n=6$ male and 6 female),

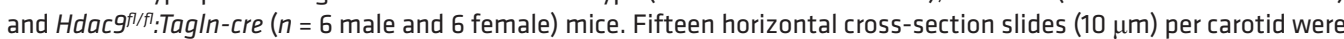
analyzed. (B) Histological and immunofluorescence analysis of ligated carotids shows that strong inhibition of neoin-

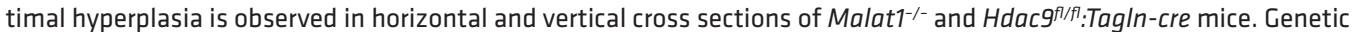
ablation of Hdac9 or Malat1 restored protein levels of Acta2 ( $\alpha$-Sma, red), Cnn1 (yellow), and Sm22 $\alpha$ (magenta) in carotid artery media. (C) Immunofluorescence analysis of ligated carotids shows preservation of the endothelial layer in

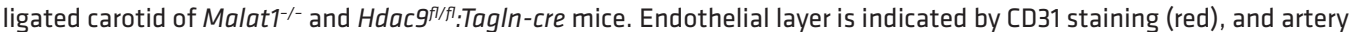
media is indicated by Acta2 staining (green) and Mmp9 (gray). Significance was calculated using 1-way ANOVA with Tukey's post hoc test. (D) Heatmap of contractile and synthetic SMC markers from ligated carotid of Malat1-- $(n=7)$ and $\mathrm{Hdac}^{f / / f}: T a g / n$-cre $(n=7)$ mice versus ligated carotid $(n=7)$ of wild-type mice. Significance was calculated using an unpaired $t$ test (2-tailed, vs. wild-type ligated carotid). Scale bars: $50 \mu \mathrm{m}$, zoom 5-fold magnification.

the complex including vascular smooth muscle transcription factors such as $M E F 2 A, M E F 2 C$, and $M Y O C D$ (Figure 1). Interestingly, one major target is the ACTA2 gene itself, consistent with previous observations of low $\alpha$-SMA levels in both aneurysms and stenotic lesions. Deficiency of either MYOCD or ACTA2 is known to exacerbate neointimal stenosis after vascular injury $(17,18)$, implicating repressive control of these gene products as a pathogenic step in neointimal stenosis. Therefore, to further explore the HDAC9-complex activity in vascular injury we used the murine carotid arterial ligation model. Consistent with the role of 

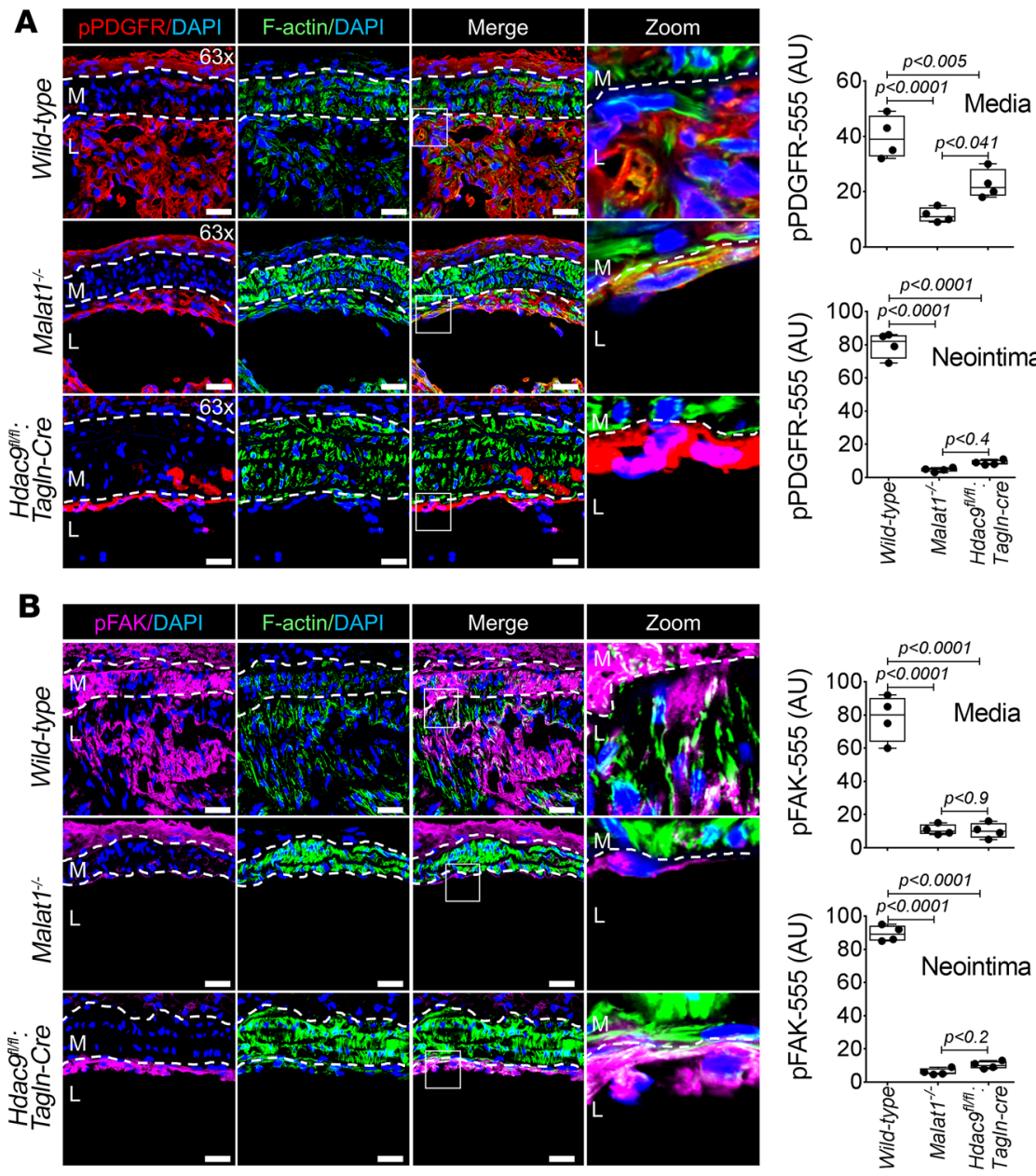

Figure 4. Genetic disruption of Hdac9 or Malat1 inhibits activation of SMC proliferation markers. (A) Immunofluorescence analysis of p-PDGFR $\beta$ (red), F-actin (green), and nuclei (blue) in ligated carotid of wild-type $(n=4)$,

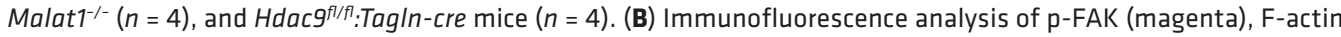

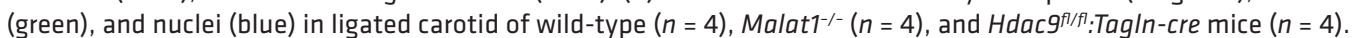
Significance was calculated using 1-way ANOVA with Tukey's post hoc test. Dashed lines indicate arterial media. M, media; L, lumen. Scale bars: $50 \mu \mathrm{m}$, zoom 5-fold magnification.

the HDAC9 complex in pathogenic progression, mice deficient in either HDAC9 or MALAT1 expression were less prone to developing neointimal hyperplasia after vascular injury and maintained contractile protein expression. We therefore explored pharmacologic targeting of the complex. First, antisense oligonucleotides targeting MALAT1 were injected into wild-type mice prior to and 1 week after carotid ligation. GapmeR-Malat1-treated animals, similar to Malat1-knockout mice, were resistant to neointimal development after carotid ligation. Next, we treated mice with the EZH2 inhibitor, GSK343, during carotid ligation. EZH2 is the catalytic subunit of PRC2, known to be recruited by the HDAC9 complex, to catalyze the trimethylation of histone $\mathrm{H} 3$ (H3K27me3) (19). Similar in effect to inhibition of MALAT1, blockage of EZH2 activity effectively inhibited neointimal formation and stenosis of the vessel with salutary effects on MMP production and contractile protein expression. These data demonstrate the feasibility of targeting epigenetic complexes necessary for neointimal development.

Arterial stenosis is a common human condition and the health impact is staggering. While the majority of arterial stenosis is associated with atherosclerosis, stenosis can also occur in the context of restenosis after percutaneous intervention, or congenital stenoses such as aortic or pulmonary obstruction. In the latter cases, obstruction is thought to be caused by excessive medial or intimal expansion of SMCs. Arterial SMCs undergo profound phenotypic changes during these disease processes, having the ability to acquire characteristics of chondrocytes, osteocytes, and macrophages (20). However, one of the most common forms is a less differentiated cellular form known as the proliferative phenotype. This phenotype is characterized 
A

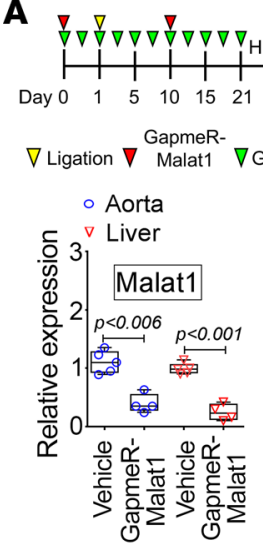

C

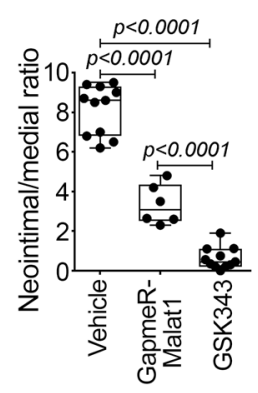

B
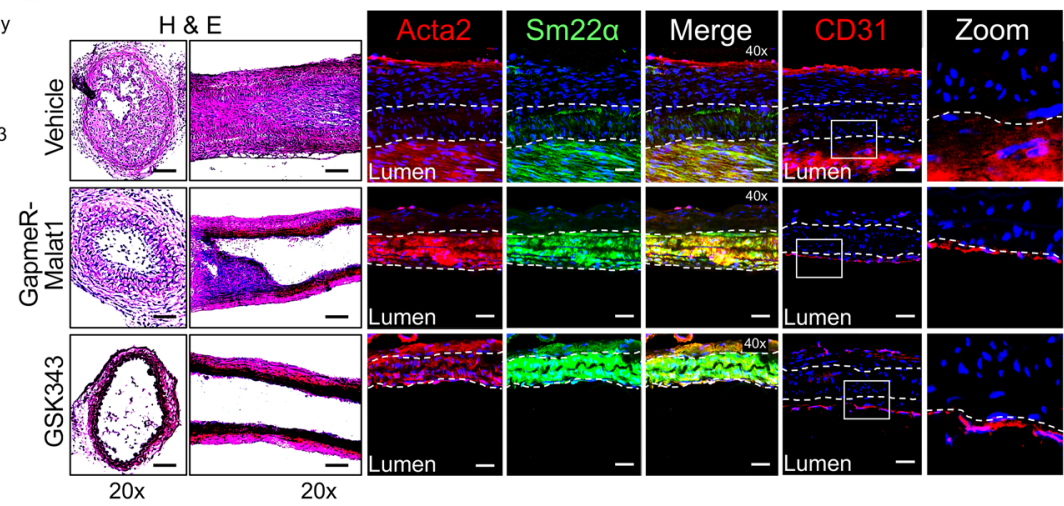

D

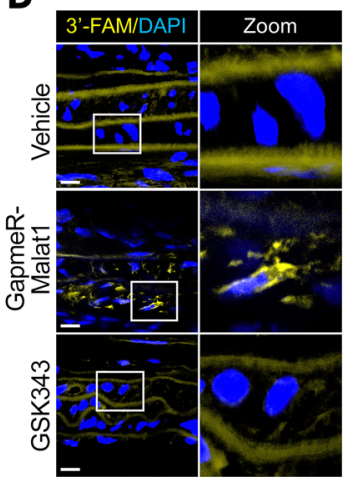

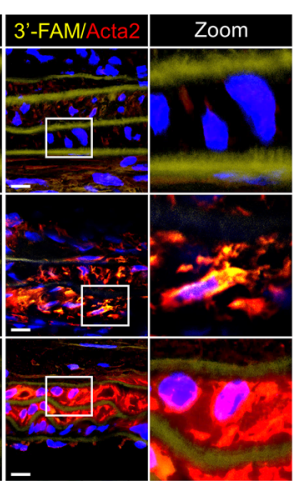

$\mathbf{E}$

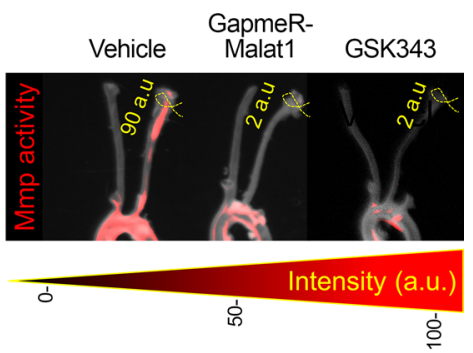

Figure 5. Pharmacologic inhibition of the Hdac9-Brg1-Malat1 complex inhibits neointimal formation. (A) Upper panel: Treatment strategy of experimental carotid artery stenosis with GapmeR-Malat1 or GSK343. Lower panel: qPCR analysis demonstrates Malat1 mRNA levels in aortic and liver tissues from vehicle- $(n=5)$ and GapmeR-Malat1-treated $(n=4)$ mice. (B) Histological and immunofluorescence analysis of ligated carotids of vehicle-, GapmeR-Malat1-, and GSK343-treated mice. Scale bars: $50 \mu \mathrm{m}$, zoom 3-fold magnification. (C) Quantification plot of vehicle ( $n=6$ male and 6 female), GapmeR-Malat1 ( $n=3$ male and 3 female), and GSK343 ( $n=6$ male and 6 female) treatment groups show significant inhibition of neointimal formation. (D) Detection of CapmeR-Malat1 molecule conjugated with 3'-FAM fluorophore in ligated carotid of GapmeR-Malat1-treated mice but not in vehicle- and GSK343-treated mice. Scale bars: $50 \mu \mathrm{m}$, zoom 5-fold magnification. (E) Inhibition of Mmp activity in ligated aortas of GapmeR-Malat1- and GSK343-treated mice. Mice were tail vein injected with MMPSense 750 FAST, a near-infrared fluorescence sensor for MMP2 and MMP9 activity, 24 hours prior to sacrifice, dissection, and imaging. Significance was calculated using 1-way ANOVA with Tukey's multiple comparisons test.

by cellular proliferation, loss of definitive markers of the SMC phenotype (such as $\alpha$-SMA), and increased activation of matrix-degrading enzymes $(2,20)$. While most arterial stenosis occurs in the context of atherosclerosis, some rare forms of Mendelian vascular smooth muscle dysfunction also encompass arterial stenosis as a phenotype. One such condition is the vascular disease caused by mutations in ACTA2. Patients with particular missense mutations in ACTA2 (and to a lesser extent in MYH11) show intracranial and coronary arterial stenosis thought to be caused by excessive medial hyperplasia $(6,21)$. These data may help inform the specific pathology seen in these rare diseases.

The transcriptional changes that occur in experimental medial hyperplasia have been well documented. In our study, we observe that the HDAC9-MALAT1-BRG1 complex targets important genes such as MEF2A, MEF2C, and MYOCD, encoding regulators of smooth muscle differentiation (Figure 1). In particular, myocardin, the master regulator of smooth muscle differentiation, has been implicated as a protective factor in neointimal stenosis (18). Mice deficient in this factor, responsible for the maintenance of VSMC identity, exhibit exacerbation of intimal reaction to arterial injury. Similarly, mice deficient in ACTA2 exhibit the same exacerbation of stenosis and intimal reaction (17). Preventing the silencing of the ACTA2 gene through inhibition of the HDAC9 complex therefore may prevent the phenotype switching necessary for neointimal proliferation. Similarly TAGLN, encoding SM $22 \alpha$, is associated with the transcriptional control of MMP expression and maintenance in expression of this gene, and may partially account for the suppression of MMP expression noted in the phenotype of Malat $^{-/-}$and Hdac fl/fl $_{\text {Tagln-cre mice. }}$ 
A

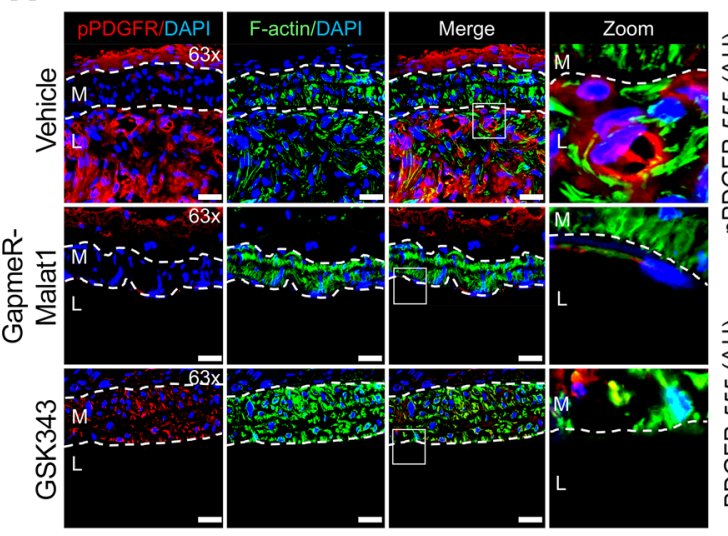

B

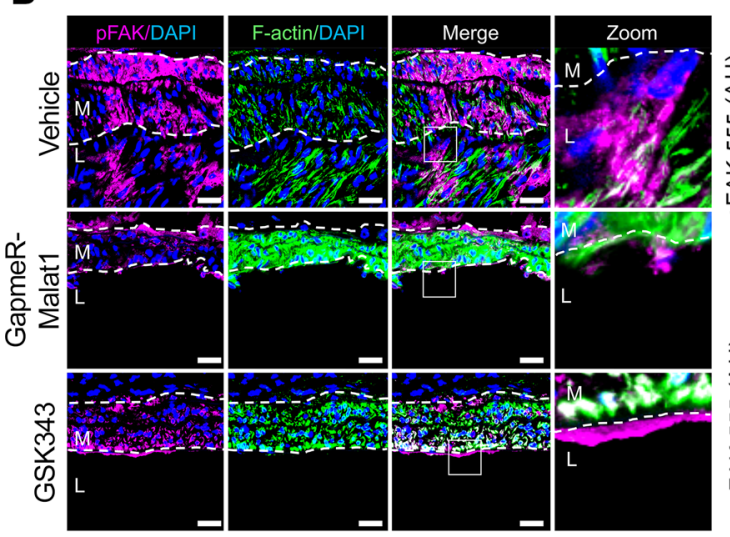

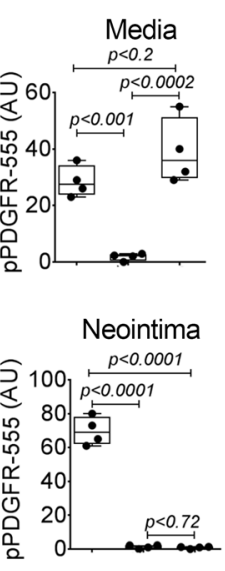

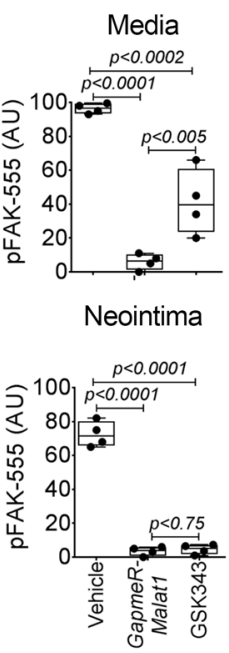

C

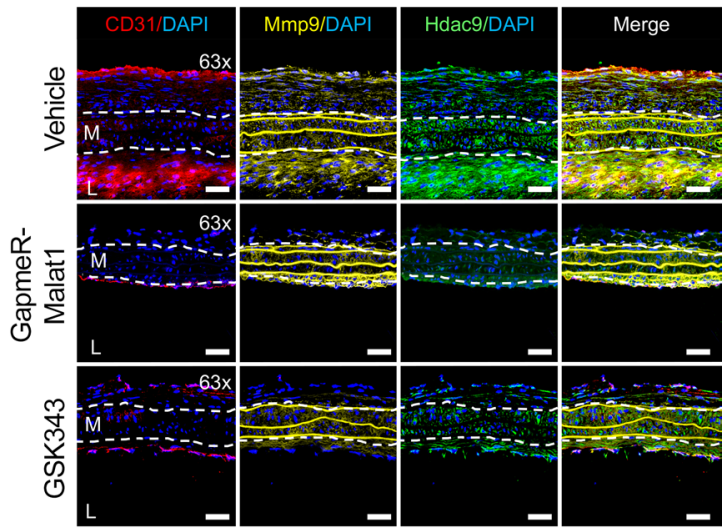

Neointima
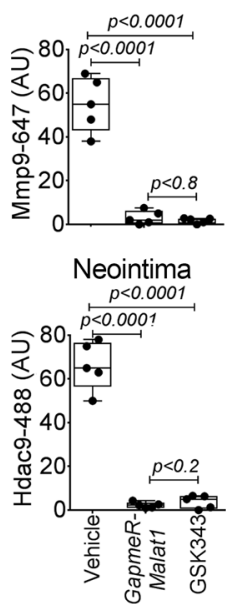

Figure 6. Pharmacologic inhibition of the Hdac9-Brg1-Malat1 complex inhibits SMC proliferation and downregulates Hdac9 expression. (A) Immunofluorescence analysis of p-PDGFR $\beta$ (red), F-actin (green), and nuclei (blue) in ligated carotid of vehicle $(n=4)$, GapmeR-Malat1 ( $n=4)$, or GSK343 ( $n=4)$ treatment groups. Scale bars: $50 \mu \mathrm{m}$, zoom 5-fold magnification. (B) Immunofluorescence analysis of p-FAK (magenta), F-actin (green), and nuclei (blue) in ligated carotid of vehicle $(n=4)$, GapmeR-Malat1 $(n=4)$, or GSK343 $(n=4)$ treatment groups. Scale bars: $50 \mu$ m, zoom 5-fold magnification. (C) Immunofluorescence analysis of CD31 (red), Mmp9 (yellow), and Hdac9 (green) in ligated carotid of vehicle ( $n=4$ ), GapmeR-Malat1 ( $n=4)$, or GSK343 ( $n=4)$ treatment groups. Significance was calculated using 1-way ANOVA with Tukey's multiple comparisons test. Dashed lines indicate arterial media. M, media; L, lumen.

Neointimal hyperplasia after vascular injury can occur in the context of stent placement for relief of arterial obstruction. The presence of the stent induces proliferation and expansion of the medial SMCs, which can cause a secondary stenosis and re-obstruct blood flow. Stents impregnated with antiproliferative agents such as rapamycin prevent neointimal proliferation. Unfortunately, the mechanism of action also prevents proper endothelialization, necessitating treatment with antiplatelet agents that increase the risk of hemorrhage (22). Agents that have the capacity to impede VSMCs without preventing endothelialization could improve this situation. Based on the data presented here, agents that inhibit the action of the HDAC9 complex may have a comparative advantage in the treatment of such stenotic vascular disease.

\section{Methods}

Mouse model of carotid stenosis. Carotid ligations were performed in 10-week-old mice. Briefly, animals were anesthetized using intraperitoneal ketamine/xylazine ( 80 and $12 \mathrm{mg} / \mathrm{kg}$, respectively) followed by a small incision in the neck to expose the carotid artery. Then the left carotid was ligated at the carotid bifurcation level using an 8-0 silk suture. At 21 days mice were sacrificed and carotids were collected for histological analysis. Approximately $98 \%$ of wild-type mice developed stenotic lesions. Mice that developed thrombosis of the ligated carotid were excluded from the study $(\sim 1 \%-2 \%)$. Malat 1 -knockout mice were provided by David Spector (Cold Spring Harbor, New York, USA). Hdac $9^{n / f}:$ Tagln-cre mice were generated as previously reported (10). $E z h 2^{f / f /}$ (stock $E z h 2^{2 m 2 S h o} / \mathrm{J}$ ) and Myh11-cre-GFP [B6.Cg-Tg(Myh11-cre,-EGFP)2Mik/J] were 
crossed to generate SMC-Ezh2-deficient mice. All the above mice and wild-type mice (C57BL/6J) were purchased from The Jackson Laboratory. For tissue analysis, animals were euthanized through inhalational isoflurane (Sigma-Aldrich) prior to tissue collection. All carotid ligation (left carotid) procedures were performed at 10 weeks of age. All experiments were performed on male and female animals at a 1:1 ratio. See complete unedited blots in the supplemental material.

Histology. Left and right carotids were cryosectioned using a standard OCT protocol and sectioning. The right carotid artery was unligated and served as an internal control. Neointimal analysis was performed from H\&E-stained horizontal cross sections embedded in OCT compound. Briefly, the distal 0.2 $\mathrm{mm}$ of carotid from the ligation suture site was discarded followed by generation of 20 slides $(10 \mu \mathrm{m})$. Slides 1, 5, 10, and 15 were H\&E stained for quantification of neointima. For quantification of the neointima, internal and external elastic lamina perimeters and medial thickness from 4 quadrants were measured and averaged using ImageJ software (NIH). Slides 2-4, 6-9, and 11-14 were used for immunofluorescence staining. All antibodies are listed in Supplemental Table 3.

$R N A$ isolation and RT-qPCR analysis. Dissected carotids were collected into a $1.5-\mathrm{ml}$ tube containing $700 \mu$ of TRIzol and total RNA was prepared using an RNeasy kit (Qiagen) following the manufacturer's protocol. The cDNA was prepared by reverse transcription (RT), and gene expression was analyzed by quantitative PCR (qPCR) on a SYBR green system (Applied Biosystems). Expression results were analyzed by the $\triangle \triangle \mathrm{CT}$ method, and GAPDH (encoding glyceraldehyde-3-phosphate dehydrogenase) was used as a housekeeping gene. Fold changes were calculated as the average relative to the control carotid as the baseline. All primers are listed in Supplemental Table 3.

In vivo MMP activity. Mice were tail vein injected with $600 \mu 1$ of MMPSense 750 FAST, a near-infrared fluorescence sensor for MMP2 and MMP9 activity (PerkinElmer). Mice were sacrificed 24 hours after injection and aortas were dissected and analyzed using a Kodak Image Station 4000MM Pro for macroscopic fluorescence reflectance molecular imaging.

Sequential FISH and immunofluorescence microscopy. Stellaris FISH Probes recognizing mouse Malat1 (SMF-3008-1) and labeled with Quasar 570-labeled oligos (Biosearch Technologies, Inc.) were hybridized to tissue samples, followed by incubation with primary and secondary antibodies following the manufacturer's instructions available online at www.biosearchtech.com/stellarisprotocols. Imaging and analysis were performed using Volocity 5.2 software. Three-dimensional and quantitative fluorescence colocalization analyses were performed as described previously (10). Two-dimensional and white-light images were analyzed using ImageJ software.

ChIP-seq and ChIP-qPCR. Human wild-type or aneurysm (ACTA2 ${ }^{\mathrm{R} 179 \mathrm{H}}$ or TGFR2 ${ }^{\mathrm{G} 357 \mathrm{~W}}$ ) (10) SMCs (10 million) were fixed with $1 \%$ formaldehyde at $37^{\circ} \mathrm{C}$ for 20 minutes, quenched with $125 \mathrm{mM}$ glycine for 5 minutes (room temperature), and protein lysates were prepared using an EpiTect ChIP kit according to the manufacturer's instructions (Qiagen). Next, total protein lysates were sonicated to shear chromatin to an average length of 500-1,000 bp, followed by centrifugation for 10 minutes at max speed. Supernatants were collected into a 2-ml tube containing $6 \mu \mathrm{g}$ of monoclonal antibody against HDAC9 (ab59718), BGR1 (ab110641), H3K27me3 (ab4729), or IgG isotype control (ab171870) (all from Abcam) and 1 $\mathrm{ml}$ of lysis buffer supplemented with $1 \times$ protease inhibitor cocktail (Roche Diagnostics), followed by incubation overnight (14 hours) at $4^{\circ} \mathrm{C}$. Immunoprecipitates were analyzed using the EpiTect ChIP kit according to the manufacturer's instructions. Then, ChIP-ed DNA was quantified and used for DNA-end repair ( 3 '-dA) followed by PCR amplification and size selection (usually 100-400 bp, including adaptor sequence). The qualified libraries were used for sequencing (Illumina HiSeq $50 \mathrm{SE}$ ). Then, fastqs files were aligned to the human genome (hg19, ENSEMBL version 75) using bowtie2 aligner (23) with up to 50 alignments reported. Reads that were multimapped, duplicated, or that contained more than 2 mismatches were filtered out using samtools v1.3.1 (24). The filtered alignment files in the form of bam files were indexed for the ability to view them on Integrative Genome Viewer (25). Peak calling was performed using Model-based Analysis for ChIP-seq version 2 (MACS2) caller between aneurysm (ACTA2 $2^{\mathrm{R} 179 \mathrm{H}}$ or TGFR2 ${ }^{\mathrm{G} 357 \mathrm{~W}}$ ) SMCs and their corresponding control sample at a $P$-value threshold of 0.05. The annotation of the peaks from MACS2 was performed using the ChipSeeker package in R (26). Briefly, the reads that were mapped to unplaced scaffolds were removed from the analysis. Before annotating the peaks with UCSC's hg19 knownGene database, the genomic coordinates were first converted from ENSEMBL to UCSC and all peaks with a transcription start site region defined at 5 kilobases of the gene location were annotated. Pathway analysis was performed using DAVID (27) with all genes 
that contained differentially expressed peaks at a $P$ value $<10^{-4}$ that were in both groups of comparisons ACTA2 $2^{\text {R179H }}$ or TGFR2 ${ }^{\mathrm{G} 357 \mathrm{~W}}$ cells versus wild-type cells. For ChIP-qPCR, human wild-type or aneurysm (ACTA2 ${ }^{\mathrm{R} 179 \mathrm{H}}$ ) SMCs at $60 \%$ confluence in a T-75 flask were transfected with siRNA targeting MALAT1 $(30 \mu \mathrm{M})$ or siRNA negative control $(30 \mu \mathrm{M})$ for 48 hours in OPTIMUM media followed by 24 hours with complete growth media. Then, cells were fixed with $1 \%$ formaldehyde at $37^{\circ} \mathrm{C}$ for 10 minutes and quenched with $125 \mathrm{mM}$ glycine for 5 minutes at room temperature, followed by preparation of total protein lysates using the EpiTect ChIP kit according to the manufacturer's instructions. Next, sheared DNA was incubated with $6 \mu \mathrm{g}$ of monoclonal antibody against H3K27me3 (Abcam, ab4729) or IgG isotype control (Abcam, ab171870). qPCR was used to analyze signals in the input and immunoprecipitates. The percentage of immunoprecipitate signals was calculated over the input signals. Experiments were performed in triplicate, with independent samples. All primers and antibodies are listed in Supplemental Table 3. All relevant data are available from the authors. Genomic data from ChiP-seq experiments were deposited in the NCBI's Gene Expression Omnibus database (GEO GSE120394).

In vivo GSK343 and Malat1 antisense LNA treatments. Cohorts of 10 -week-old wild-type mice were treated with GSK343 in drinking water 24 hours before left carotid ligation and following treatment for 3 weeks. GSK343 inhibitor $(25 \mathrm{mg}$ ) was dissolved in $1 \mathrm{ml}$ of DMSO to obtain a stock solution of $46 \mathrm{mM}$. Then, $250 \mathrm{ml}$ of drinking water was supplemented with $250 \mu 1$ of GSK343 stock solution. The control group was given equal volumes of sterile water. Bottles containing GSK343 were replaced with fresh water supplied with drug every 3 days. For Malat1 antisense locked nucleic acid (LNA) treatments, cohorts of 10-week-old wild-type mice were injected with $15 \mathrm{nmol}$ in saline solution 24 hours before left carotid ligation followed by a second injection 7 days after ligation.

Statistics. Results are given as mean \pm SD. Student's $t$ test (2-tailed) was applied to determine the statistical significance of differences between control and treated groups. $P$ less than 0.05 was considered statistically significant $\left({ }^{*} P<0.05,{ }^{* *} P<0.01\right.$, and $\left.{ }^{* *} P<0.001\right)$. For all experiments, at least 3 experimental replicates were performed. Scatter graphs show mean $\pm \mathrm{SD}$. One-way analysis of variance (ANOVA) was used to analyze histology data involving multiple mouse genotypes ( $95 \%$ confidence interval is plotted). $P$ values represent 1-way ANOVA followed by Tukey's honestly significant difference (HSD) post hoc test. All graphs were produced using GraphPad Prism 7.0.

Study approval. All experimental procedures were approved by the Institutional Animal Care and Use Committee (IACUC) of the MGH. Patient imaging data were shared under protocol approved by the Partners HealthCare IRB after informed written consent (protocol 2000P001531).

\section{Author contributions}

MEL and CLLC conceptualized the study. MEL, CWK, CLLC, NLW, and FAJ developed the methodology. CLLC, CWK, EC, and BG conducted the investigation. MEL, RM, and FAJ provided resources. CLLC and ASY curated the data. MEL and CLLC wrote the original draft of the manuscript. MEL, SD, NLW, and FAJ acquired funding.

\section{Acknowledgments}

MEL is supported by the Fredman Fellowship, the Toomey Fund for Aortic Dissection Research, and the Hassenfeld Fellowship. MEL and CLL are supported by NIH grant HL130113. NLW is supported by NIH grants HL126949, HL112640, HL134354, and AR070029. RM is supported by NIH grant K08HL111210, the Hassenfeld Fellowship, and the Wild Family Foundation. EC is supported by the MGH cardiovascular sciences T32 training grant. FAJ and CWK are supported by NIH grant HL137913.

Address correspondence to: Mark E. Lindsay, Harvard Medical School, Richard B. Simches Research Building, Room 3212, 185 Cambridge Street, Boston, Massachusetts 02114, USA. Phone: 617.643.3458; Email: Lindsay.Mark@mgh.harvard.edu.

1. Ailawadi G, et al. Smooth muscle phenotypic modulation is an early event in aortic aneurysms. J Thorac Cardiovasc Surg. 2009;138(6):1392-1399.

2. Alexander MR, Owens GK. Epigenetic control of smooth muscle cell differentiation and phenotypic switching in vascular development and disease. Annu Rev Physiol. 2012;74:13-40.

3. Guo DC, et al. Mutations in smooth muscle alpha-actin (ACTA2) lead to thoracic aortic aneurysms and dissections. Nat Genet. 
2007;39(12):1488-1493.

4. Pannu H, et al. MYH11 mutations result in a distinct vascular pathology driven by insulin-like growth factor 1 and angiotensin II. Hum Mol Genet. 2007;16(20):2453-2462.

5. Milewicz DM, et al. Genetic basis of thoracic aortic aneurysms and dissections: focus on smooth muscle cell contractile dysfunction. Annu Rev Genomics Hum Genet. 2008;9:283-302.

6. Guo DC, et al. Mutations in smooth muscle alpha-actin (ACTA2) cause coronary artery disease, stroke, and Moyamoya disease, along with thoracic aortic disease. Am J Hum Genet. 2009;84(5):617-627.

7. Milewicz DM, et al. De novo ACTA2 mutation causes a novel syndrome of multisystemic smooth muscle dysfunction. Am $J$ Med Genet A. 2010;152A(10):2437-2443.

8. Keylock A, et al. Moyamoya-like cerebrovascular disease in a child with a novel mutation in myosin heavy chain 11 . Neurology. 2018;90(3):136-138.

9. Huang J, et al. Myocardin is required for maintenance of vascular and visceral smooth muscle homeostasis during postnatal development. Proc Natl Acad Sci USA. 2015;112(14):4447-4452.

10. Lino Cardenas CL, et al. An HDAC9-MALAT1-BRG1 complex mediates smooth muscle dysfunction in thoracic aortic aneurysm. Nat Commun. 2018;9(1):1009.

11. Lino Cardenas CL, et al. Inhibition of the methyltranferase EZH2 improves aortic performance in experimental thoracic aortic aneurysm. JCI Insight. 2018;3(5):97493.

12. International Stroke Genetics Consortium (ISGC), et al. Genome-wide association study identifies a variant in HDAC9 associated with large vessel ischemic stroke. Nat Genet. 2012;44(3):328-333.

13. CARDIoGRAMplusC4D Consortium, et al. Large-scale association analysis identifies new risk loci for coronary artery disease. Nat Genet. 2013;45(1):25-33.

14. Zhang B, et al. The lncRNA Malat1 is dispensable for mouse development but its transcription plays a cis-regulatory role in the adult. Cell Rep. 2012;2(1):111-123.

15. Owens LV, et al. Overexpression of the focal adhesion kinase (p125FAK) in the vascular smooth muscle cells of intimal hyperplasia. J Vasc Surg. 2001;34(2):344-349.

16. Ferns GA, Raines EW, Sprugel KH, Motani AS, Reidy MA, Ross R. Inhibition of neointimal smooth muscle accumulation after angioplasty by an antibody to PDGF. Science. 1991;253(5024):1129-1132.

17. Papke CL, et al. Smooth muscle hyperplasia due to loss of smooth muscle $\alpha$-actin is driven by activation of focal adhesion kinase, altered p53 localization and increased levels of platelet-derived growth factor receptor- $\beta$. Hum Mol Genet. 2013;22(15):3123-3137.

18. Talasila A, et al. Myocardin regulates vascular response to injury through miR-24/-29a and platelet-derived growth factor receptor- $\beta$. Arterioscler Thromb Vasc Biol. 2013;33(10):2355-2365.

19. Boyer LA, et al. Polycomb complexes repress developmental regulators in murine embryonic stem cells. Nature. 2006;441(7091):349-353.

20. Owens GK, Kumar MS, Wamhoff BR. Molecular regulation of vascular smooth muscle cell differentiation in development and disease. Physiol Rev. 2004;84(3):767-801.

21. Georgescu MM, et al. The defining pathology of the new clinical and histopathologic entity ACTA2-related cerebrovascular disease. Acta Neuropathol Commun. 2015;3:81.

22. Buccheri D, Piraino D, Andolina G, Cortese B. Understanding and managing in-stent restenosis: a review of clinical data, from pathogenesis to treatment. J Thorac Dis. 2016;8(10):E1150-E1162.

23. Langmead B, Salzberg SL. Fast gapped-read alignment with Bowtie 2. Nat Methods. 2012;9(4):357-359.

24. Li H, et al. The Sequence Alignment/Map format and SAMtools. Bioinformatics. 2009;25(16):2078-2079.

25. Robinson JT, et al. Integrative genomics viewer. Nat Biotechnol. 2011;29(1):24-26.

26. Yu G, Wang LG, He QY. ChIPseeker: an R/Bioconductor package for ChIP peak annotation, comparison and visualization. Bioinformatics. 2015;31(14):2382-2383.

27. Huang DW, et al. DAVID Bioinformatics Resources: expanded annotation database and novel algorithms to better extract biology from large gene lists. Nucleic Acids Res. 2007;35(Web Server issue):W169-W175 